\title{
Thermal Radiation from Photonic Crystals: A Direct Calculation
}

\author{
Chiyan Luo, Arvind Narayanaswamy, Gang Chen, and J. D. Joannopoulos* \\ Center for Materials Science and Engineering and Department of Mechanical Engineering, Massachusetts Institute of Technology, \\ Cambridge, Massachusetts 02139, USA
}

(Received 20 April 2004; published 19 November 2004)

\begin{abstract}
A classical simulation of equilibrium thermal emissivity from dispersive, lossy photonic crystals is presented. Normal emission results consistent with those assuming Kirchoff's law are obtained; i.e., a photonic crystal does not emit more than what a blackbody does. Significant enhancement, however, can be achieved over the radiation intensity from a uniform slab, indicating the potential usefulness of photonic crystals in incandescent lighting and thermal photovoltaic applications.
\end{abstract}

DOI: 10.1103/PhysRevLett.93.213905

PACS numbers: 42.70.Qs

Thermal radiation has been a subject of interest since the pioneering work at the beginning of the last century due to Planck [1-3]. Recently, the thermal radiation from complex electromagnetic structures known as photonic crystals [4-6] has attracted attention [7-13]. Most notably, intriguing experiments by Lin et al. $[11,12]$ indicated that the thermal radiation from metallic photonic crystals may even exceed that of a blackbody in free space. With regard to that phenomenon, the possibility of thermal nonequilibrium during the measurements has also been acknowledged $[14,15]$. It is the purpose of the present Letter to show that this phenomenon does not occur under the assumption of thermal equilibrium. Nevertheless, with a given material, the level of emission from a photonic crystal can be closer to that of a blackbody than from a uniform slab.

Previously, Trupke et al. [16] questioned the validity of interpreting the results of Lin et al. as exceeding the blackbody limit with a thermodynamic argument. Here we study the thermal emission from photonic crystals directly by performing numerical simulation on photonic crystals made from absorbing and highly dispersive materials in dimensions higher than one, under the assumption of fluctuation-dissipation theorem. Our simulation indicates that the ensemble-averaged equilibrium radiation emissivity from such photonic crystals is indeed equal to their absorbance. Our results also show, however, that significant enhancement can be achieved over the radiation from a uniform slab, indicating the potential usefulness of such structures in incandescent lighting and thermal photovoltaic applications in addition to their spectral selectivity, as suggested in the original work by Lin et al.

The method we use to model the thermal electromagnetic emission is a finite-difference time-domain (FDTD) approach incorporating the well-established Langevin approach to Brownian motion [17,18]. In the absence of thermal agitations, the dispersive and absorbing materials are modeled by a harmonic polarization response $\mathbf{P}$ to the local electric field $\mathbf{E}$ :

$$
\frac{d^{2} \mathbf{P}}{d t^{2}}+\gamma \frac{d \mathbf{P}}{d t}+\omega_{0}^{2} \mathbf{P}=\sigma \mathbf{E}
$$

with $\mathbf{P}=n e \mathbf{r}$ and $\sigma=n e^{2} / m$. Here $e$ is the charge carried by the microscopic particles responsible for the polarization response (electrons in the case of a metal), with mass $m$ and number density $n, \gamma$ is the frictional coefficient expressing the losses in the system, and $\omega_{0}$ is the resonance frequency of the polarization system $\left(\omega_{0}=0\right.$ in the Drude model for metals). The Langevin approach consists of adding a random term $\mathbf{K}(t)$ representing thermal fluctuations on the electromagnetic field to the righthand side of Eq. (1). The response of the system then reads as

$$
\frac{d^{2} \mathbf{P}}{d t^{2}}+\gamma \frac{d \mathbf{P}}{d t}+\omega_{0}^{2} \mathbf{P}=\sigma \mathbf{E}+\mathbf{K}(t) .
$$

Equations (1) and (2) can be discretized in the standard way [19] by $d^{2} \mathbf{P} / d t^{2} \approx[\mathbf{P}(t+\delta t)-2 \mathbf{P}(t)+\mathbf{P}(t-$ $\delta t)] / \delta t^{2}$ and $d \mathbf{P} / d t \approx[\mathbf{P}(t+\delta t)-\mathbf{P}(t-\delta t)] /(2 \delta t)$, and $\mathbf{P}(t+\delta t)$ can then be updated in terms of $\mathbf{P}(t)$ and $\mathbf{P}(t-\delta t)$. Once the random force term $\mathbf{K}(t)$ is specified, Eq. (2) can then be integrated in time to simulate the fluctuating thermal radiation. In terms of the conventional FDTD algorithm, this procedure means updating $\mathbf{P}$ before updating $\mathbf{E}$ and adding $\mathbf{P}$ to $\mathbf{E}$ through $\mathbf{E}(t+$ $\delta t)=\mathbf{E}(t)+4 \pi[\mathbf{P}(t)-\mathbf{P}(t+\delta t)] / \epsilon_{\infty}$, where $\epsilon_{\infty}$ is the medium permittivity in the absence of polarizations, before updating the magnetic field $\mathbf{H}$ [19].

Here, we note that a white-noise spectrum in $\mathbf{K}$, corresponding to a classical high-temperature behavior, may be used in a classical FDTD algorithm to simulate the thermal emissivity at all temperature levels. To see this, let us look at the correlation function of $\mathbf{K}$ in the frequency domain. When applied independently everywhere within the polarization response, $\mathbf{K}$ produces fluctuations $\mathbf{Q}(\mathbf{r}, \omega)=4 \pi \mathbf{K}(\mathbf{r}, \omega) /\left(\omega_{0}^{2}-\omega^{2}-i \gamma \omega\right)$ in the displacement fields at spatial position $\mathbf{r}$ and angular frequency $\omega$ satisfying the following fluctuation-dissipation relation, as derived by Rytov [2]:

(C) 2004 The American Physical Society

213905-1 


$$
\begin{aligned}
\left\langle Q_{i}(\mathbf{r}, \omega) Q_{j}^{*}\left(\mathbf{r}^{\prime}, \omega^{\prime}\right)\right\rangle= & \frac{16 \pi^{3} c^{2} \operatorname{Im}[\epsilon(\omega)]}{\omega^{3}} \\
& \times I_{0}(\omega, T) \delta_{i j} \delta_{\omega \omega^{\prime}} \delta\left(\mathbf{r}-\mathbf{r}^{\prime}\right),
\end{aligned}
$$

where $Q_{i}$ for $i=1,2,3$ are the components of $\mathbf{Q},\langle\cdots\rangle$ denotes ensemble averaging, $c$ is the speed of light, $\operatorname{Im}[\epsilon(\omega)]$ is the imaginary part of the permittivity including the polarization response in the absence of fluctuations [Eq. (1)], and $I_{0}(\omega, T)=\frac{c}{4 \pi} D(\omega) \mathcal{E}(\omega, T)$ is the free space Planck blackbody radiation intensity. $D(\omega)=$ $\frac{\omega^{2}}{\pi^{2} c^{3}}$ is the free-space density of photon states and $\mathcal{E}(\omega, T)=\hbar \omega /[\exp (\hbar \omega / k T)-1]$ is the Bose-Einstein energy distribution function at absolute temperature $T$. The appearance of $I_{0}(\omega, T)$ in Eq. (3) manifests the universality of the thermal fluctuations: it is consistent with Planck's formula and Kirchoff's law in analytical geometries including dielectrics [2]. In the discrete limit, the fluctuations in $\mathbf{K}$ thus become

$$
\left\langle K_{i}(\mathbf{r}, \omega) K_{j}^{*}\left(\mathbf{r}, \omega^{\prime}\right)\right\rangle=\frac{4 \pi^{2} \sigma \gamma \delta_{i j} \delta_{\omega \omega^{\prime}}}{\Delta V} \frac{I_{0}(\omega, T)}{(\omega / c)^{2}},
$$

where $\Delta V$ is the volume element used in the simulation. To obtain emissivity, the target thermal emission intensity needs to be normalized by that of the free-space Planck radiation. The linearity of the system ensures that this normalization procedure amounts to dividing the right-hand side of Eq. (4) by the Planck radiation collected within the emission solid angle. For a solid angle element $d \Omega$ around a specific direction that makes an angle $\theta$ with the $z$ axis, the Planck intensity is $I_{0}(\omega, T) \cos \theta d \Omega=I_{0}(\omega, T) d k_{x} d k_{y} /(\omega / c)^{2}$, where $k_{x}$ and $k_{y}$ are the wave vectors along the $x$ and $y$ axes. When we employ an FDTD algorithm with Bloch-periodic boundary conditions in the $x y$ plane and perfectly matched layers boundary conditions [19] along the $z$ sections, the directions are specified by $k_{x}$ and $k_{y}$ at frequency $\omega$, and the normalization factor is thus proportional to $I_{0}(\omega, T) /(\omega / c)^{2}$. Therefore, for emissivity calculations, all frequency-dependent factors on the right-hand side of Eq. (4) are effectively removed, and we can replace $\mathbf{K}(\mathbf{r}, \omega)$ by a white-noise spectrum $\mathbf{K}^{\prime}(\mathbf{r}, \omega)=$ $C^{\prime} \mathbf{K}(\mathbf{r}, \omega) \sqrt{(\omega / c)^{2} / I_{0}(\omega, T)}$, where $C^{\prime}$ is a dimensioncorrecting factor that depends only on the discretization details of the system. In the time domain, the autocorrelation function of $\mathbf{K}^{\prime}(\mathbf{r}, t)$ is given by $\left\langle K_{i}^{\prime}(\mathbf{r}, t) K_{j}^{\prime *}\left(\mathbf{r}, t^{\prime}\right)\right\rangle=$ $\frac{1}{N^{2}} \sum \omega \omega^{\prime}\left\langle K_{i}^{\prime}(\mathbf{r}, \omega) K_{j}^{\prime *}\left(\mathbf{r}, \omega^{\prime}\right) e^{-i \omega t+i \omega^{\prime} t^{\prime}}\right\rangle=\frac{4 \pi^{2} C^{\prime 2}}{N \Delta V} \sigma \gamma \delta_{i j} \delta_{t t^{\prime}}$, with $N$ being the number of time steps in the Fourier transform. The time series of the normalized excitation $\mathbf{K}^{\prime}$ can thus be sampled simply as independent random drawings from a distribution law with variance $\left\langle\left|K_{i}^{\prime}(\mathbf{r}, t)\right|^{2}\right\rangle$.

We have performed FDTD numerical simulations in the above framework for thermal radiation from photonic crystals made of metals whose dielectric function [Eq. (1)] is $\epsilon(\omega)=\epsilon_{\infty}-\sigma /\left(\omega^{2}+i \gamma \omega\right)$ ( $\sigma$ here equals
$4 \pi \sigma$ in the above analysis). We have obtained the normal emissivity under excitations of $\mathbf{K}^{\prime}$ by calculating the radiation flux through planes parallel to slab surfaces for various two-dimensional (2D) and 3D crystal geometries [20], with lateral periodic boundary conditions. We have used the following simple uniform probability distribution density for the independent drawings of $\mathbf{K}^{\prime}$ :

$$
w\left[K_{i}^{\prime}\right]=\left\{\begin{array}{cl}
1 / K_{s} & \text { if }\left|K_{i}^{\prime}\right|<K_{s} / 2 \\
0 & \text { if }\left|K_{i}^{\prime}\right|>K_{s} / 2
\end{array}\right.
$$

with $K_{s}^{2}=C \sigma \gamma$. The discretization-specific constant $C=48 \pi^{2} C^{2} /(N \Delta V)$ can be eliminated by a set of calibration runs in which the photonic crystal is replaced by a 1D uniform slab. Since for a 1D slab the emissivity is known analytically to equal its absorbance, the results from the calibration provide the conversion factor to translate flux into emissivity for all structures at the same computational resolution. A comparison between the emissivity calculated in this way can then be made to the absorbance of the same structure, which is calculated separately without the use of fluctuating fields. We find this comparison among 1D/2D/3D structures to be quite similar. Since computations in two dimensions are much more tractable than in three, we were able to perform 2D calculations and average over many statistical samples. For better accuracy we will focus on some typical results in two dimensions, in particular, for a square lattice of metallic rods. The simulated radiation shows a highly fluctuating behavior, and the ensembleaveraged results are shown in Fig. 1. On average, the thermal emissivity spectrum agrees with the absorption spectrum to an excellent degree, clearly demonstrating that the photonic crystal is not expected to emit more than that from a blackbody. The main features in the emission/absorption spectrum can be explained by known spectral properties of metallic photonic crystals [21,22], and we have noted the frequency regions in which photonic band gaps are expected for the crystal studied here in Fig. 1. In the case of interest here, $\gamma$ is small and $\sigma$ is large compared to $2 \pi c / a$, and an optically thick uniform metallic slab is both a poor absorber and a poor emitter. The absorbance tends to $\sqrt{8 \omega \gamma / \sigma}$ as $\omega \rightarrow 0$ and is limited by approximately $2 \gamma / \sqrt{\sigma}$ when $\gamma<\omega<\sqrt{\sigma}$. This uniform-slab value is determined by the metallic surfaces only and is independent of the slab thickness. On the other hand, the emissivity of metallic photonic crystals exceeds that of the uniform slab for almost all frequencies. When the frequency falls within a photonic propagating band of the crystal, emission inside the whole crystal bulk can escape to the free space and contribute to the total emission. The metallic photonic crystal exhibits lower values of emissivity when the frequency falls within a photonic band gap, but the value is still higher than that of a uniform slab, owing to a larger penetration depth (a smaller effective $\sigma$ ) on the crystal surface. The only case that such a metallic photonic crystal emits less 
than a uniform slab is in the TE case when $\omega \rightarrow 0$, as this is in the low-loss limit for a dielectriclike behavior of the photonic crystal. The absorption/radiation properties in this limit is determined by the product of the imaginary part of the effective dielectric constant, which is a harmonic mean of those of the crystal components, and a scaling factor of $L / \lambda$, where $L$ is the thickness of the crystal slab accounting for light propagation through the bulk crystal. This gives rise to an emissivity scaling such as $\omega^{2}$ and can become less than those of a uniform slab as $\omega \rightarrow 0$. The frequency region in the propagating bands of the crystal where the emission significantly exceeds that of a uniform metallic slab by a factor of 3 to 4 is the case of practical interest for lighting source applications with
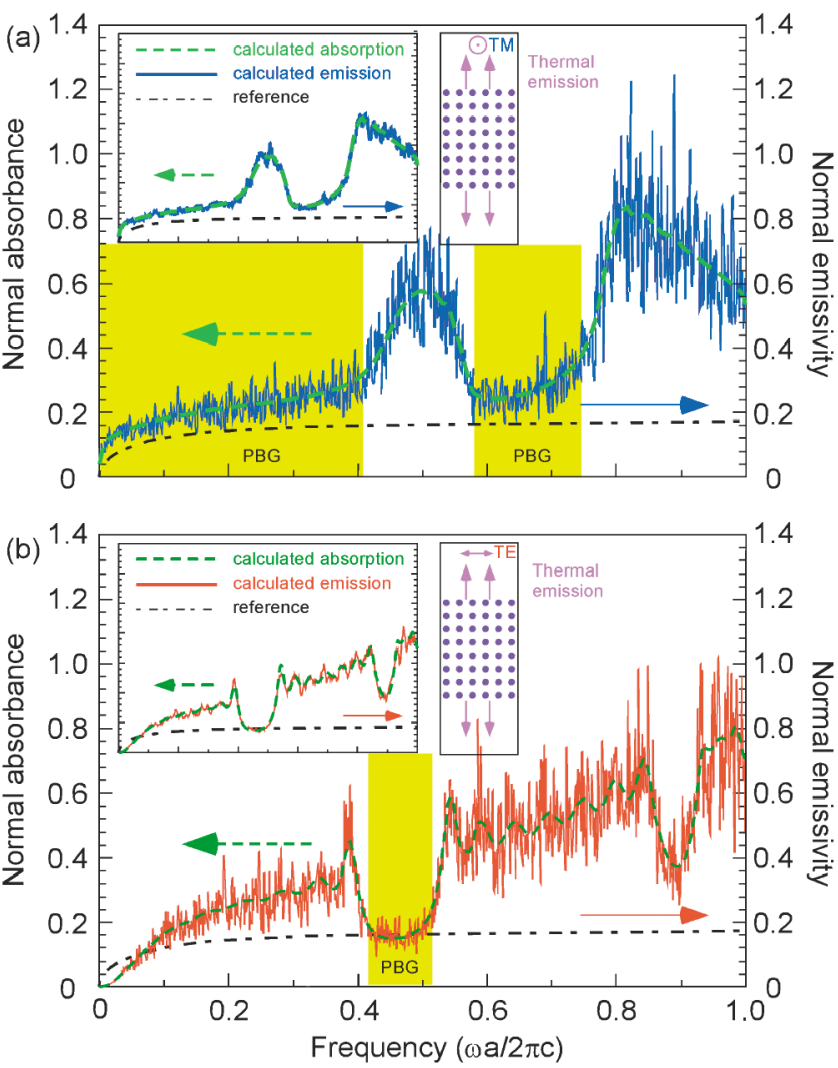

FIG. 1 (color). Comparison between the absorption and thermal emission from a $2 \mathrm{D}$ photonic crystal from a direct calculation. The crystal is eight layers of metal rods in a square lattice (lattice constant $a$, discretized by 40 grid points) with radii $r=0.2 a$ shown in the insets. The parameters for the metal are $\epsilon_{\infty}=1, \gamma=0.3(2 \pi c / a)$, and $\sigma=10\left(4 \pi^{2} c^{2} / a^{2}\right)$. The light propagation direction is normal to the interfaces and the light is polarized (a) along the rods (TM) and (b) perpendicular to the rods (TE). Each simulation iterates through $N=80000$ time steps, corresponding to a total time of $1000 \mathrm{a} / \mathrm{c}$. The emission curves are averaged results over 40 independent simulations, and results further averaged over a frequency interval $\Delta \omega=0.01(2 \pi c / a)$ containing ten frequency points are shown in the insets. The reference curve is the absorption spectrum for a uniform metallic slab with the same thickness. The frequency ranges of photonic band gaps (PBGs) are highlighted in yellow. such materials, particularly in the infrared and visible part of the spectrum. Except in the low-loss dielectric behavior as discussed above, such an enhancement effect should be a general phenomenon that does not require a complete photonic band gap and can occur in metallic systems when extended electromagnetic states exist. Thus, for example, a quasicrystalline or random array of small metallic rods/wires is expected to emit much more light compared to a uniform metallic slab. This is to be contrasted with the existing enhancement techniques due to surface plasmons [23] or surface roughening, in which the improvement occurs mainly on the surface instead of throughout the bulk of the material.

We have also performed further calculations for different material parameters, and the results are shown in Fig. 2. As $\sigma$ becomes smaller the features in the emissivity spectrum due to photonic crystal effect becomes weaker and less distinct, and the shapes of the spectrum change accordingly. At all levels of $\sigma$, however, the ensemble-averaged thermal emissivity is seen to follow the variations in the absorbance in all aspects. These calculations therefore directly confirm that the expected radiation intensity $\langle I(\omega, T)\rangle$ for this photonic crystal is related to the free-space blackbody radiation intensity $I_{0}(\omega, T)$ by the absorbance $A(\omega)$ :

$$
\langle I(\omega, T)\rangle=A(\omega) I_{0}(\omega, T) ;
$$

i.e., Kirchoff's law also holds for absorbing and highly dispersive photonic crystal structures. Note that in Eq. (6) the effects due to the photonic density of states of the complex structure are fully captured through $A(\omega)$. Therefore, in the thermal equilibrium situation studied here, the only possible deviations of the emissivity from absorbance are purely due to statistical fluctuations. If we regard the thermal emission studied here as originating from a photon gas residing in the photonic crystal at thermal equilibrium, we can infer the fluctuations in the emission intensity from the well-known Einstein expression for fluctuations in the occupation number $n$ of thermal photons at temperature $T$ that states [24] $\left\langle\Delta n^{2}\right\rangle /\langle n\rangle^{2}=1 /\langle n\rangle-a$, where $a=-1$ in the BoseEinstein case corresponding to wave nature of light, and $1 /\langle n\rangle$ accounts for the radiation's particle nature (not included in our simulation). A statistical analysis on our ensemble data directly gives $\sqrt{\left\langle\Delta I(\omega, T)^{2}\right\rangle}=$ $\langle I(\omega, T)\rangle$ with a quantitative comparison similar to that shown in Fig. 2, and thus our simulation also faithfully reproduces the wave component of thermal fluctuations in the emission. The expression for the physical fluctuations in thermal radiation from photonic crystals is given by

$$
\sqrt{\left\langle\Delta I(\omega, T)^{2}\right\rangle}=\exp \left(\frac{\hbar \omega}{2 k T}\right)\langle I(\omega, T)\rangle .
$$

It is thus evident that photonic crystals can be used to strongly modify not only the expectation value of thermal radiation intensity but also the magnitude of thermal fluctuations. 


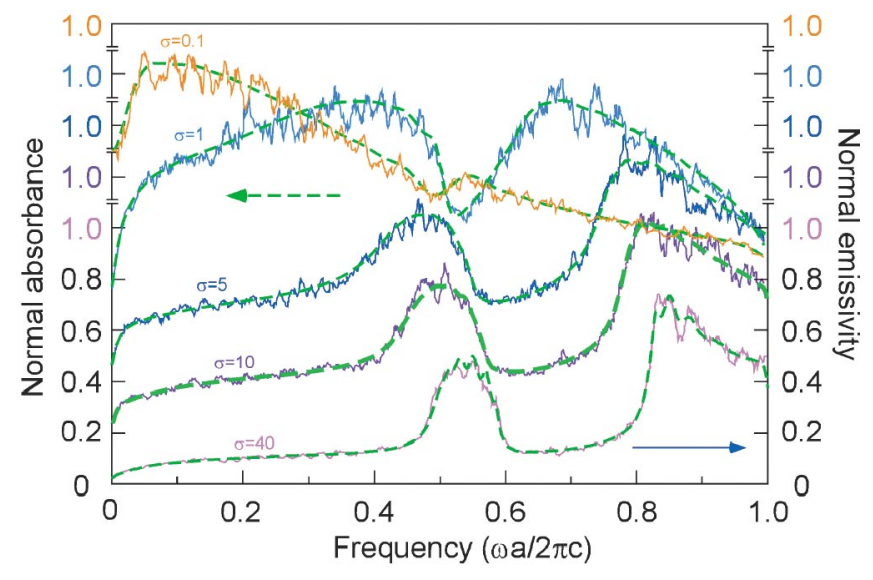

FIG. 2 (color). Further comparison between thermal emissivity from a direct calculation (averaged over 40 samples and ten frequency points) and absorbance for various $\sigma$ 's. The polarization is TM in all cases. For clarity, all curves are up-shifted by 0.2 in absorbance ( 1 in emission flux) from each other as $\sigma$ decreases. The $\sigma$ 's are in units of $4 \pi^{2} c^{2} / a^{2}$.

In this work we have determined, through a direct calculation, the equilibrium thermal emission properties of a photonic crystal system. The results support the traditional indirect method [8] of calculating absorbance and assuming Kirchoff's law, and the thermal radiation from photonic crystals in equilibrium can exceed only that of a blackbody by means of fluctuations. Our simulation is built naturally on the basis of Brownian motion theory, and there are at least three reasons why such an approach is interesting as compared to the traditional indirect method. First, this treatment brings out the statistical nature of thermal emission directly and gives detailed information on the fluctuations in the thermal emission in the classical limit. Such fluctuations are the essence of thermal phenomena, but they are not directly available from the indirect method. Second, this approach is not limited to the specific configuration considered here and can be applied in a straightforward manner to find equilibrium thermal emission in arbitrary geometries including all solid angles. Except in a few analytical geometries, similar calculations required by the indirect method would be very difficult or tedious to carry out. Third, the present approach is physically general and can incorporate the case of thermodynamic nonequilibrium and transient phenomena. The case of nonequilibrium steady state [25], for example, is a particularly interesting case in which the fluctuating field $\mathbf{K}$ is no longer governed by the fluctuation-dissipation relation [Eq. (3)] but is determined by external driving conditions. Such a case is of course beyond the bounds of the indirect method, for which thermal equilibrium is a necessary assumption to start with.

We thank our colleagues David Roundy, Elefteris Lidorikis, Xunya Jiang, Peter Bermel, Marin Soljačić, and Ivan Celanovic at the Massachusetts Institute of
Technology for helpful discussions. This work was supported in part by the Material Research Science and Engineering Center program of the National Science Foundation under Grant No. DMR-0213282, the Department of Defense (Office of Naval Research) Multidisciplinary University Research Initiative program under Grant No. N00014-01-0803, and the U.S. Department of Energy under Grant No. DE-FG02-99ER45778.

*Electronic address: joannop@mit.edu

[1] M. Planck, The Theory of Heat Radiation (Dover, New York, 1959).

[2] S. M. Rytov, Theory of Electric Fluctuations and Thermal Radiation (Academy of Sciences Press, Moscow, Russia, 1953), English translation 1959 ed.

[3] J. B. Pendry, J. Phys. Condens. Matter 11, 6621 (1999).

[4] E. Yablonovitch, Phys. Rev. Lett. 58, 2059 (1987).

[5] S. John, Phys. Rev. Lett. 58, 2486 (1987).

[6] J. D. Joannopoulos, R. D. Meade, and J. N. Winn, Photonic Crystals: Molding the Flow of Light (Princeton University Press, Princeton, NJ, 1995).

[7] P. Pigeat, D. Rouxel, and B. Weber, Phys. Rev. B 57, 9293 (1998).

[8] C. M. Cornelius and J. P. Dowling, Phys. Rev. A 59, 4736 (1999).

[9] Z.-Y. Li, Phys. Rev. B 66, 241103(R) (2002).

[10] S.-Y. Lin, J. G. Fleming, E. Chow, J. Bur, K. K. Choi, and A. Goldberg, Phys. Rev. B 62, R2243 (2000).

[11] S.-Y. Lin, J. Moreno, and J. G. Fleming, Appl. Phys. Lett. 83, 380 (2003).

[12] S.-Y. Lin, J. G. Fleming, and I. El-Kady, Opt. Lett. 28, 1909 (2003).

[13] A. Narayanaswamy and G. Chen, Phys. Rev. B 70, 125101 (2004).

[14] S.-Y. Lin, J. Moreno, and J. G. Fleming, Appl. Phys. Lett. 84, 1999 (2004).

[15] S.-Y. Lin, I. El-Kady, and G. S. Subramania (private communication).

[16] T. Trupke, P. Wurfel, and M. A. Green, Appl. Phys. Lett. 84, 1997 (2004).

[17] S. Chandrasekhar, Rev. Mod. Phys. 15, 1 (1943).

[18] C. H. Henry and R. F. Kazarinov, Rev. Mod. Phys. 68, 801 (1996).

[19] A. Taflove and S. C. Hagness, Computational Electrodynamics: The Finite-Difference Time-Domain Method (Artech House Publishers, Boston, 2000), 2nd ed.

[20] The geometries we have investigated include 2D square and triangular lattices of rods in air and holes in dielectric as well as 3D woodpile structures.

[21] V. Kuzmiak, A. A. Maradudin, and F. Pincemin, Phys. Rev. B 50, 16835 (1994).

[22] M. M. Sigalas, C. T. Chan, K. M. Ho, and C. M. Soukoulis, Phys. Rev. B 52, 11744 (1995).

[23] M. U. Pralle et al., Appl. Phys. Lett. 81, 4685 (2002).

[24] R. K. Pathria, Statistical Mechanics (ButterworthHeinemann, Oxford, UK, 1996), 2nd ed.

[25] M. Lax, Rev. Mod. Phys. 32, 25 (1960). 